\title{
Universal Usability statements: Marking the trail for all users
}

\section{Harry Hochheiser and Ben Shneiderman}

Draft: December 31, 2000 -- do not circulate --

\section{Introduction}

Signposts at ski areas help skiers to choose slopes that match their skills and equipment. They describe easy, intermediate and expert trails, indicate which ones are groomed, and give their length or vertical drop. Skiers use this information to make informed decisions, avoiding routes that may be too difficult or too long. They may choose different skis or waxes depending on the conditions.

For web surfers, good guidance and safe visits are still too rare. Despite extensive research and improved products, the primary experience of many users is frustration and anxiety. Common problems include long download times, incompatible browser versions, and unavailable plug-ins. Too often users find that their screen size does not match the designer's screen size, needed fonts are not installed, or media players just don't work on their machine. These problems are troubling to experienced users as well as novices and even more problematic to users with disabilities.

However, web site designers can take immediate measures to help web surfers find safe trails and have more successful web experiences. Just as park rangers provide information that describes the terrain and the obstacles that may be lurking around the corner, web site designers can provide web versions of this information. When it becomes apparent that too many trails are marked as 'extremely difficult', thoughtful park rangers will recognize the need to cut some more 'moderate' and 'easy' trails to enable more visitors to enjoy the scenery.

\section{Rationale}

Universal usability statements are declarations by web site designers of the usability measures and concerns associated with a given web site. The statements describe the contents of a site, browser requirements, network requirements, and other characteristics that may influence its usability.

For users, these declarations will enable them to avoid the frustration of dealing with sites that require new plug-ins, fresh browser versions, or media capabilities that are not installed. By knowing what is needed for a successful web site visit, they will be more likely to have a satisfying and successful experience. For users with disabilities, these benefits can be even more significant. For users with visual impairments, knowing that a given site has been tested with their screen reader software will ease fears of difficulty and encourage them to explore the site.

For web site operators, these statements offer a powerful means of building user confidence. The presence of a universal usability statement on a site tells users that the developers of the site are concerned about more than just showing off their prowess with the latest technology: they are concerned about the quality of the user experience. Eventually, the presence of a "Universal Usability" graphic on the site might provide a visible reminder of the availability of information about the usability of the site, just as "works best with Foo browser, version 7.9" icons currently tell users what they need to be successful site visitors. Concerned developers can also use these statements to guide revisions that will increase usability - entries in the statement that present potential usability problems can be used to build "to-do" lists for future site updates. 


\section{Universal Usability Statement Template}

Like well-written web site privacy policies, usability statements should be designed to provide as much information as possible as quickly as possible. This goal can be accomplished through the use of common phrasing and organization that will make the content of these statements easy to understand.

The universal usability template can be used to generate statements that share a common style. Starting from the basic template, web site developers can provide the information needed and trim items that do not apply. The template can be found at the universalusability.org web site.

(http:/www.universalusability.org/template.html). To demonstrate usage, an example universal usability statement for that site is available at http://www.universalusability.org/about/uupolicy.html.

The template is divided into several categories, with examples of specific entries under each category:

Basic System Requirements: Which systems has the site been tested on?

Browser Requirements: Which browsers, including version numbers? Does the site use frames? Java? Cascading Style Sheets? ActiveX Component? Which plug-ins are necessary?

Input devices: Does the site require any particular mice? Can the site be navigated with keyboard only? Which (if any) speech recognition products can be used with the site?

Display: What are the recommended and minimum screen resolutions? Was the site designed for a fixed width? What screen sizes have been tested? Mobile devices? Hand-held devices? Which font sizes have been used and tested? Do non-text messages have text equivalents?

Audio-Video: What sorts of audio and video (if any) are used? Are alternative descriptions provided?

Network Connection: What is the maximum download size for a page? What is the minimal bandwidth needed? Recommended? Which bandwidths have been tested?

Access for users with disabilities: Is there a text-only version of the site? Which alternate browsers have been tested? Which screen readers? Has the site accessibility been validated? If so, to what level?

Diverse Users: Which languages are supported? Has the site been tested on left-right and right-left languages? What education level is required for the site? Has novice user testing been conducted? Are there any other assumptions of user background?

User Support: What is the availability and response rate of online (Email) assistance? Phone assistance? Are there any online communities on the site? Is there a site FAQ? 
Contact Information: How can the webmaster be contacted? When was the

statement last updated?

These categories and questions are designed to provide users with information needed to plan their route - if the statement indicates obstacles such as an inappropriate font size or language, the user may decide to move on to another site, or - at the very least - she will be aware of the potential difficulties that lay ahead. Other users might use the statement to plan activities: upon learning that a site requires a $640 \times 480$ screen, a mobile user browsing on a handheld device might decide to wait until returning to his 21 -inch display at the office before proceeding further.

Some developers will find that many of these items do not apply to their sites. Others may find that they do not have the resources to conduct all of the testing that would be needed to address all of the items in the template. This is to be expected - universal usability is a goal to be achieved gradually. The World Wide Web Consortium (W3C) provides a useful guide to designers for prioritizing improvements in its Checklist for Web Content Accessibility (http://www.w3.org/TR/WAI-WEBCONTENT/full-checklist). However, the lack of completely universal accessibility should not stop developers from providing usability statements, as any information provided may help improve the experience of site visitors. The universal usability statements can also be used as starting points to guide improvements in usability and elimination of obstacles.

The National Institute of Standards and Technology has organized an industry group that has developed a Common Industry Format (http://www.nist.gov/iusr ) for reporting usability study results to companies procuring software that could possibly form the basis of reporting on testing with users for the universal usability template.

\section{The Future}

Web site operators interested in promoting universal usability can (and should) use the template to develop usability statements and place them prominently on their sites. Eventually, we hope to see usability statements as a standard component of site design, just as privacy policies are currently found on commercial and non-commercial sites.

To make this happen, we need your help. Publishing universal usability statements for web sites is the obvious first step. Feedback and input regarding the template is also very helpful: what's missing? What should change? What should the format be? Of course we recognize the limitations and difficulties in having a compact yet comprehensive universal usability template. It places some additional pressure on web site operators, but the payoff may come from increased user satisfaction.

Finally, and most importantly, we need champions and evangelists, individual and institutional, who will promote the idea of the universal usability statement in particular, and universal usability in general.

Responsible ski area managers prepare accurate and up-to-date ski trail information so that visitors have successful experiences. They take into consideration the needs of novices, elderly, and even disabled users. Web site developers and managers will also produce more successful and satisfying visits if they mark the trail for web users, making sure that every visitor can have a safe visit. 